\title{
La percezione e la stima del tempo in bambini di terza elementare: dall'intuizione alla consapevolezza dell'aspetto soggettivo'
}

\section{The perception and estimation of time in third grade children: from intuition to awareness of the subjec- tive aspect}

\author{
Carlo Mina
}

Istituto scolastico di Locarno - Solduno, Svizzera

Sunto / In una realtà in cui i ritmi di vita sono sempre più frenetici, cerchiamo con insistenza di avere sotto controllo ogni minuto della nostra giornata; a dipendenza delle attività che svolgiamo tuttavia, il tempo sembra durare in eterno o esaurirsi in un attimo, e lo stesso vale per i nostri allievi. Quali sono i fattori che influenzano la percezione del tempo nei bambini? È possibile attivare una consapevolezza sulla soggettività del tempo vissuto dai bambini attraverso il lavoro in classe? Lo scopo di questo lavoro è sondare le capacità di stima delle durate in un gruppo di allievi e sensibilizzarli sull'aspetto soggettivo della percezione temporale. L’esperienza, durata all'incirca due mesi, ha coinvolto 20 allievi di terza elementare in un lavoro continuo di riflessione e stima della durata di attività ludico-didattiche. Attraverso una serie di colloqui individuali, la redazione di questionari e la discussione con i compagni è stato possibile raccogliere le interessanti considerazioni dei bambini, che offrono un singolare quadro del tema dal loro punto di vista.

Parole chiave: percezione del tempo; metacognizione; stima; grandezze e misure.

\begin{abstract}
In a reality where the rhythms of life are more and more frenetic, we try to keep every minute of our day under control; depending on the activities we carry out, however, time seems to last forever or to run out in a blink, and the same applies to our students. Which are the factors that influence the perception of time in children? Is it possible to activate an awareness of the subjectivity of time experienced by children through classroom work? The aim of the research is to explore the capacity of estimating durations of a group of students and to make them aware of the subjective aspect of the perception of time. The experience, which lasted about two months, involved 20 third grade students in a continuous work of reflection and estimation of the duration of recreational and didactic activities. Through a series of individual interviews, the drafting of questionnaires and discussion with peers it was possible to collect the interesting considerations of the children, which offer a unique picture of the theme from their point of view.
\end{abstract}

Keywords: time perception; metacognition; estimation; dimensions and measures.

\section{Introduzione}

Questo contributo intende ampliare e rendere spendibile in classe un percorso sperimentato in relazione ad un lavoro di tesi bachelor svolto nel 2015 presso la scuola elementare di Minusio. La tesi intendeva sondare l'aspetto, poco approfondito in

1. Questo articolo rappresenta una sintesi del lavoro di diploma Bachelor of Arts in Insegnamento per il livello elementare di Carlo Mina (2015). La tesi è scaricabile all'indirizzo http://tesi.supsi.ch/57/. 
La percezione e la stima del tempo in bambini di terza elementare: dall'intuizione alla consapevolezza dell'aspetto soggettivo / Carlo Mina

letteratura e raramente considerato nella nostra realtà scolastica, della percezione e della stima del tempo in bambini di terza elementare, rispondendo alle seguenti domande di ricerca:

D1. Come sono le percezioni e la stima della durata di allievi di terza elementare che non hanno mai lavorato in maniera specifica sugli aspetti psicologici legati al tempo? In particolare, quali fattori (socio-affettivi, ambientali ecc.) influenzano la loro percezione e stima del tempo?

D2. Come evolve negli allievi la percezione e la stima della durata dopo un costante lavoro di sensibilizzazione con strumenti di misura diversi? In particolare, quali fattori vengono riconosciuti dagli allievi dopo un percorso di sensibilizzazione sulla soggettività della percezione temporale?

Le giornate di ogni bambino sono suddivise e organizzate secondo i criteri temporali tipici della nostra società, quindi la conoscenza del tempo e delle sue caratteristiche dovrebbe rappresentare un elemento fondamentale nello sviluppo del bambino. Paradossalmente tuttavia, gli aspetti legati alla dimensione temporale vengono spesso trattati in maniera superficiale dalla realtà scolastica, che si limita a fornire agli allievi gli strumenti per misurare il tempo e per sapersi collocare temporalmente all'interno della giornata.

Durante la sperimentazione si è potuto implementare un approccio didattico volto a sollecitare maggiormente gli aspetti legati alle capacità di stima e percezione del tempo, con il fine di rendere consapevoli gli allievi sui più elementari meccanismi psicologici che entrano in gioco quando si ragiona sulla durata di un'attività.

La speranza è quella che il percorso riesca a suscitare l'interesse del lettore rispetto al tema oggetto di studio e, perché no, che l'esperienza possa essere adattata e riproposta in altre classi. Nonostante la loro natura prettamente qualitativa, i risultati emersi dalla sperimentazione possono dare alcune indicazioni rispetto alle possibili abilità degli allievi e alle possibilità di riflessione e approfondimento che si possono mettere in atto in classe. ${ }^{2}$

Il contributo prevede una prima parte d'inquadramento teorico, una sintesi della ricerca svolta e infine una descrizione delle possibili attività da svolgere in classe.

Figura 1

Una rappresentazione spontanea degli strumenti di misurazione del tempo.

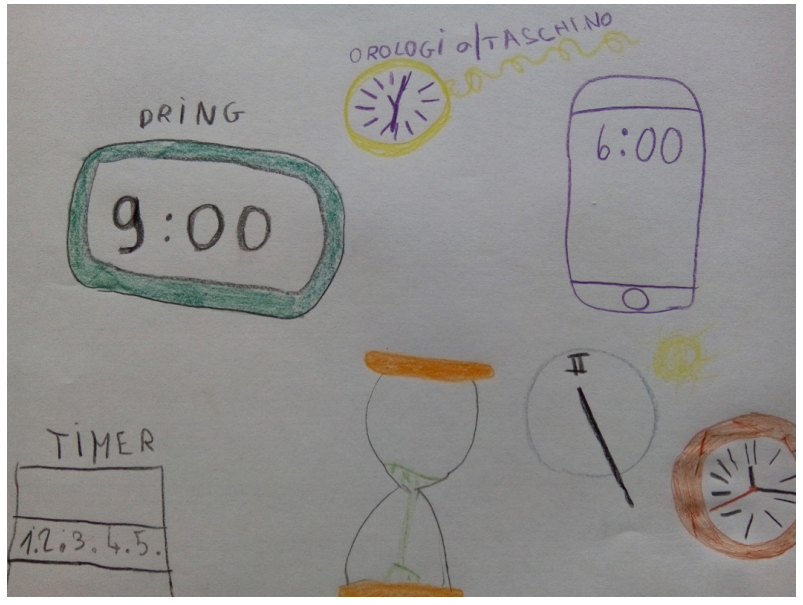

2. Si vedano i risultati emersi all'indirizzo http://tesi.supsi.ch/57/. 


\title{
2 Quadro Teorico
}

Nel 1928, un incontro tra Albert Einstein e Jean Piaget spinse lo psicologo ad occuparsi, tra gli altri innumerevoli temi, anche della percezione del tempo nel bambino. I suoi studi, durati una quindicina d'anni e raccolti nel libro "Lo sviluppo della nozione di tempo nel bambino", sono ad oggi i più dettagliati e completi sul tema. Partiamo dunque da questo autore.

Il primo termine che è opportuno analizzare è proprio quello di tempo, che per Piaget rappresenta

\begin{abstract}
«una nozione intellettuale che si costruisce in modo graduale, parallelamente sia al costruirsi della rappresentazione di un mondo esterno ordinato (...) sia alla conquista sempre più consapevole da parte del soggetto della propria identità».
\end{abstract}

(Sandri, 2007, p. 20)

Piaget sostiene, dunque, che il concetto di tempo si sviluppa nel bambino in maniera graduale, come una successione di stadi consecutivi. Tuttavia, la rappresentazione dello sviluppo del bambino in stadi è stata largamente messa in discussione dagli studi di Annette Karmiloff-Smith, che ha proposto un modello di sviluppo a fasi che, allo stato attuale, risulta più attuale e moderno.

Andando più in profondità su tale concetto, Piaget considera il tempo come la coordinazione dei movimenti; visione che è stata criticata da Jaques Montangero nel 1997, secondo il quale è possibile definire il tempo come «la dimensione del cambiamento (...). È infatti possibile distinguere completamente il tempo dallo spazio in cambiamenti come le variazioni di temperatura o di stati psicologici» (Sandri, 2008, p. 39). In questo modo Montangero arriva a dissociare dunque completamente il concetto di tempo da quello di spazio.

Piaget, basandosi sul presupposto citato in precedenza, ovvero che il tempo sia la coordinazione dei movimenti, sostiene l'esistenza di un tempo operatorio, costituito da rapporti di successione e di durata di tipo oggettivo, che si differenzia dal tempo intuitivo, nel quale l'individuo opera in maniera soggettiva e percettiva sugli aspetti legati alla successione e alla durata.

In particolare, l'autore sostiene che il tempo intuitivo non sia sufficientemente affidabile, in quanto fortemente influenzato dalla soggettività dell'individuo, e necessiti per forza l'apporto di operazioni metriche e qualitative per costruire in maniera efficace relazioni di tipo temporale. È quindi opportuno analizzare come sviluppa il bambino questa nozione di tempo, considerando dapprima la nozione di ordine e successione degli eventi e in seguito quella di durata degli intervalli.

In ambito di ordine degli eventi, Piaget (1979) afferma che il bambino, prima di poter padroneggiare la costruzione della serie irreversibile degli avvenimenti, ovvero prima di essere in grado di ordinare una successione di eventi dal primo che si è realizzato all'ultimo, deve forzatamente disporre della reversibilità del pensiero. II bambino infatti non è in grado, prima di possedere tale abilità, di riordinare sequenze di azioni osservate in precedenza.

Approfondiamo ora il concetto di durata. Pea (2001) ha proposto una definizione che esplicita in maniera chiara il significato di questo termine: 
La percezione e la stima del tempo in bambini di terza elementare: dall'intuizione alla consapevolezza dell'aspetto soggettivo / Carlo Mina

«La durata è la grandezza degli intervalli di tempo, cioè del tragitto di tempo che viene percorso: fra due istanti; fra due eventi, cioè dalla fine del primo evento all'inizio del secondo; nello svolgersi di un'azione, cioè dall'inizio di un'azione alla sua fine; nello svolgersi di un evento».

(Pea, 2001, p. 69)

Piaget (1979) sostiene che il bambino sviluppi questa nozione in tre stadi consecutivi. Nel primo stadio c'è un'assenza totale del concetto di durata e per il bambino

«la nozione stessa di durata o di intervallo di tempo resta priva di significato preciso. Questo avviene perché il bambino tende ad associare i concetti di "prima" e "dopo" all'ordine spaziale, considerando che ciò che si muove più rapidamente impiegherà automaticamente meno tempo. Questo si verifica perché il bambino non padroneggia ancora il rapporto inverso tra il tempo e la velocità: più in fretta $=$ meno tempo».

(Piaget, 1979, p. 40)

Il bambino quindi si limita a trarre delle conclusioni sulla durata di un evento mettendo la stessa in rapporto con la velocità con cui una determinata azione si compie. Nel secondo stadio il bambino comincia a considerare lo scorrere del tempo durante una determinata azione e intuisce che il rapporto tra distanza compiuta e durata non è per forza corretto, ma non riesce comunque ad applicare in maniera costante quest'intuizione.

Il passaggio dal secondo al terzo stadio avviene quando i bambini cominciano a rendersi conto che è necessario mettere in relazione la successione degli eventi con la durata degli intervalli. Quando questo avviene il bambino riesce finalmente a dissociare I'ordine spaziale da quello temporale. I problemi riscontrati fino a questo punto trovano dunque una soluzione e il bambino riesce a costruire una nozione di tempo che comprende tutti gli eventi e coordina durata e ordine di successione. Questi presupposti permettono al bambino di apprendere a manipolare un'unità di tempo e questo gli consente di misurare delle durate. Per misurare il tempo, Piaget (1979) sostiene che

«il bambino (...) deve comprendere che l'orologio non cambia velocità e può indicare i tempi successivi uguali; che il tempo dell'orologio è identico a quello dei movimenti o delle azioni da cronometrare e che lo spazio percorso dalla sabbia o dalla lancetta può essere diviso in unità di tempo uguali tra loro nella successione e applicabili alla durata degli altri movimenti».

(Piaget, 1979, p. 197)

Qualcuno potrebbe chiedersi, in fondo, se per il bambino la padronanza assoluta del concetto di durata sia così importante. Un'interessante teoria in merito è quella di Pea (2001), che sostiene che la padronanza di tale nozione sia per il bambino un vero e proprio bisogno, che scaturisce soprattutto dall'interazione con i compagni:

"L'interagire in contemporaneità con i compagni, costringe il bambino a confrontare le sue azioni con le azioni degli altri. È questo raffronto che porta il bambino a giudicare la propria azione con una nuova grandezza: la durata».

(Pea, 2001, p. 69) 
La percezione e la stima del tempo in bambini di terza elementare: dall'intuizione alla consapevolezza dell'aspetto soggettivo / Carlo Mina

Piaget (1979), nel suo approfondito studio, sostiene inoltre che il bambino debba poi riuscire a riconoscere due eventi della medesima durata e individua anche in questo ambito gli stessi tre stadi citati in precedenza: nel primo stadio il bambino non è in grado di riconoscere né la simultaneità, né il sincronismo di due eventi e non riesce nemmeno a quantificare l'azione che è avvenuta; nel secondo stadio il bambino scopre il rapporto inverso del tempo e della velocità e comincia a padroneggiare il concetto di simultaneità, ma solo in seguito riesce a sincronizzare le durate di due eventi; nel terzo ed ultimo stadio il bambino arriva a padroneggiare la sincronizzazione e la quantificazione dell'azione ed è dunque in grado di uguagliare le durate sincrone.

Contro tale visione si è schierata Iris Levin, che ha criticato la teoria sviluppata da Jean Piaget in quanto le prove che ha effettuato erano, a suo avviso, troppo complesse per i bambini. Levin (1982) sostiene infatti che proponendo prove diverse e meno complesse sia possibile riconoscere nei bambini capacità logiche superiori a quelle mostrate dai lavori di Piaget.

Arriviamo ora al nodo della questione, ovvero al tema della percezione delle durate nel bambino. Piaget dedica un intero capitolo del suo libro a tale proposito e lo intitola "Il tempo vissuto". Questa definizione trasmette in maniera molto immediata I'idea che la percezione del passare del tempo, per il bambino come per l'adulto, sia in realtà assolutamente dipendente dal vissuto della persona, ovvero da quello che il soggetto svolge, sente, assimila e vive durante lo scorrere del tempo. L'autore sostiene che la durata interiore di un'azione non sia altro che il tempo dell'azione così come viene vissuta dall'individuo, ovvero la relazione tra il soggetto e gli oggetti su cui agisce.

Piaget ha poi individuato due categorie di fattori che influenzano la durata vissuta durante un'azione: i fattori periferici (quali lo sforzo, la velocità, i risultati materiali delle azioni ecc.) e i fattori centrali, ovvero della coscienza pura del tempo. In questo ambito Piaget (1979) scopre una

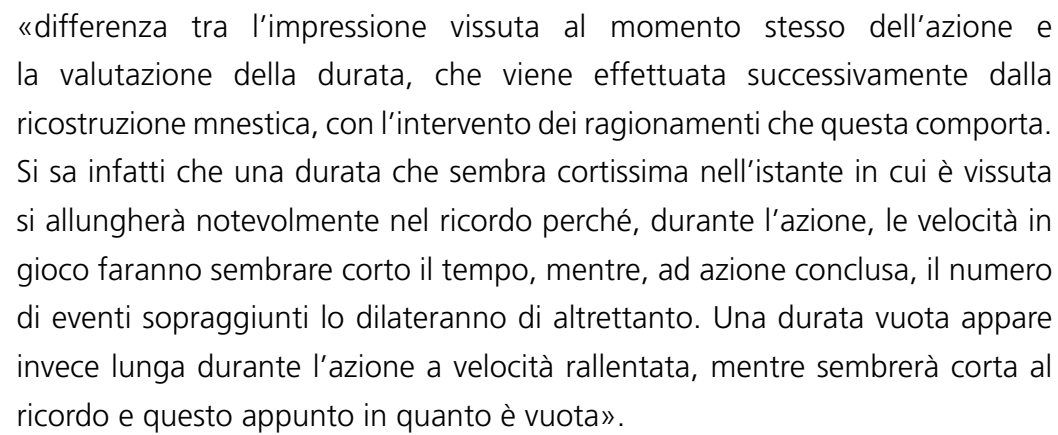

(Piaget, 1979, p. 242)

In particolare Piaget ha intuito quanto la percezione del tempo fosse influenzata da alcuni fattori: egli insiste sulla differenza tra tempo dell'attesa e tempo dell'interesse e sul rapporto tra la valutazione delle durate e la difficoltà d'esecuzione dell'azione svolta. Per quanto riguarda il tempo dell'attesa e della noia, gli studi di Piaget hanno dimostrato come il $100 \%$ dei bambini consideri il tempo in cui si annoia o deve aspettare più lungo di quello in cui si diverte.

Anche Cardaci (2004) individua tre fattori che influiscono sulla percezione del tempo, quali il livello di soddisfazione del vissuto, il livello di prevedibilità del vissuto e I'orizzonte temporale in cui il vissuto si colloca. Le variabili che sono state studiate 
La percezione e la stima del tempo in bambini di terza elementare: dall'intuizione alla consapevolezza dell'aspetto soggettivo / Carlo Mina

e che influenzano la percezione del tempo nel bambino sono sforzo, soddisfazione, interesse, orizzonte temporale, risultati, prevedibilità, velocità (categorizzati da Piaget come "fattori periferici") e coscienza (categorizzata da Piaget come "fattore centrale") (Tabella 1).

Tabella 1

Riassunto delle variabili presenti in letteratura che influenzano la percezione del tempo.

\begin{tabular}{|l|l|}
\hline Variabili che influenzano la percezione & Categorizzazione Piagettiana \\
\hline Sforzo - Difficoltà dell'azione svolta & Fattori periferici \\
\hline Soddisfazione - Livello di soddisfazione relativo al vissuto & \\
\hline Interesse - Livello di noia o divertimento percepito & \\
Orizzonte temporale - Quando ha avuto luogo l'azione? & \\
\hline Risultati - Risultati ottenuti dall'azione & \\
\hline Prevedibilità - Livello di prevedibilità relativo al vissuto & \\
\hline Velocità - La velocità con cui tale azione viene svolta & Fattori centrali \\
\hline Coscienza - La coscienza oggettiva del tempo & \\
\hline
\end{tabular}

Ovviamente, le variabili fin qui citate sono state individuate dagli autori in situazioni di ricerca non per forza legate alla scuola, dunque è possibile che altri fattori, soprattutto di tipo periferico, siano presenti e influiscano sulla percezione temporale dei bambini.

L'opera di Piaget, nelle sue pagine finali, trae una conclusione interessante che contestualizza bene questo lavoro:

«Nei più piccoli, il carattere egocentrico, ossia immediato e irreversibile, del pensiero è un ostacolo ad ogni introspezione: la presa di coscienza dell'azione propria inizia dunque con quella dei suoi risultati e soltanto in seguito si risale con un duplice sforzo di inversione rispetto a questo orientamento iniziale e di decentramento o confronto, alla coscienza del meccanismo stesso di tale azione».

(Piaget, 1979, p. 266)

Le considerazioni di Piaget portano a ipotizzare un'incapacità degli allievi di scuola elementare nel riflettere, in maniera introspettiva, sugli aspetti che influenzano la percezione del tempo. In realtà, oggi si ipotizza che i bambini dispongano di abilità riflessive più sviluppate, ed è per questa ragione che durante la sperimentazione sono state messe alla prova la loro capacità di riconoscere alcuni fattori che influiscono sulla percezione del tempo e soprattutto la loro capacità di considerare questi aspetti come soggettivi e diversi da bambino a bambino.

Tale sperimentazione non verte però soltanto sull'aspetto della percezione del tempo e sul riconoscimento dei fattori che influenzano tale percezione, ma anche sulla stima delle durate di attività di tipo scolastico. Calvani (1988) afferma che la stima del tempo nella vita quotidiana può assumere tre forme: «un giudizio/sentimento assoluto "lungo", "corto"; un confronto "più/meno lungo di..."; una valutazione che si appoggia alle unità convenzionali» (p. 63).

Secondo Fraisse (1975), quando l'individuo cerca di stimare una durata senza rife- 
La percezione e la stima del tempo in bambini di terza elementare: dall'intuizione alla consapevolezza dell'aspetto soggettivo / Carlo Mina

rimenti temporali o sistemi di misura, tenta di ricordare la quantità di cambiamenti che si sono susseguiti durante il periodo di tempo da stimare. Anche lo stesso Fraisse tuttavia riconosce che sia la percezione, sia la memorizzazione dei cambiamenti avvenuti durante un determinato lasso di tempo, sono due atti cognitivi che vengono influenzati dai fattori esterni che abbiamo elencato in precedenza. Tale idea è appoggiata anche da Michon (1979), che aggiunge tra i fattori determinanti nella stima di una durata, oltre al numero delle attività svolte, anche la loro complessità. Piaget (1979) approfondisce tali riflessioni sostenendo che l'individuo che stima la durata di un intervallo agisce nella maniera seguente:

«Si ha dapprima l'illusione come tale, ossia l'errore sistematico che fa giudicare un tempo vissuto lungo o corto a seconda di certi fattori di attività e di tensione interiore. Vi è poi la reazione a questa illusione, nel senso di un'accettazione precritica del dato percettivo o, al contrario, di una progressiva correzione che si effettua mediante un gioco di regolazioni dapprima, e, alla fine, di confronti operatori. Si ha, in terzo luogo, e questo è fondamentale, la differenza tra l'impressione vissuta al momento stesso dell'azione e la valutazione della durata, che viene effettuata successivamente dalla ricostruzione mnestica, con I'intervento dei ragionamenti che questa comporta».

(Piaget, 1979, p. 242)

Questo significa che la percezione della durata rappresenta per l'individuo un'illusione, in quanto questa è stata influenzata da una serie di fattori di cui abbiamo già parlato in precedenza. Quest'illusione può essere consapevole, e in questo caso il soggetto cercherà di correggere la sua stima in funzione dei fattori che ha riconosciuto, oppure inconsapevole, e in questo caso l'individuo si limiterà ad accogliere senza spirito critico il dato percettivo. Ciò che però accomuna chiunque si trovi nella situazione di dover stimare una durata è che tra la percezione vissuta nel momento dell'azione e la successiva valutazione della sua durata, vi è sempre una differenza. Attività di stima della durata d'intervalli di tempo brevi vengono proposte da Sandri (2008a), la quale sostiene che con questi lavori è possibile perseguire, sin dalla scuola dell'infanzia, obiettivi quali il saper riconoscere l'inizio e la fine di un dato evento; fare stime, inizialmente in modo soggettivo e in seguito sulla base dell'osservazione di strumenti di misura quali clessidre o candele, relative alla durata delle principali attività della vita scolastica. Durante lo svolgimento di queste attività è opportuno utilizzare strumenti di misura diversi, alcuni tipici del mondo adulto, altri più intuitivi e comprensibili da parte del bambino, come le clessidre ad acqua o a sabbia o le candele a tacche.

In un estremo tentativo di sintesi è possibile affermare che lo sviluppo della nozione di tempo nel bambino avviene in maniera graduale. II bambino deve dapprima impossessarsi delle capacità logiche per padroneggiare i concetti temporali, come pure della reversibilità del pensiero. Una volta raggiunto quest'obiettivo, il bambino s'impadronisce delle nozioni di successione, di contemporaneità, di simultaneità e infine di durata. Questa nozione di durata considera due aspetti complementari: quello del tempo psicologico e quello del tempo fisico. In entrambi i casi è possibile definire la durata di un periodo, utilizzando però modalità diverse. Nel caso del tempo psicologico, l'individuo ipotizza la durata di un intervallo in maniera soggettiva, cercando di ricostruire il corso degli eventi e operando sotto l'influsso di fattori che ne modificano la percezione; nel caso del tempo fisico invece, attraverso l'utilizzo di 
strumenti di misurazione, è possibile determinare la durata effettiva ed oggettiva di un determinato periodo.

\section{Sperimentazione didattica}

\subsection{Le finalità}

Il percorso svolto aveva quali finalità principali la riflessione sugli aspetti soggettivi che influenzano la percezione del tempo; lo sviluppo delle capacità di stima di una durata e lo sviluppo delle abilità di introspezione relative alle proprie capacità di stima.

Tale lavoro, legato principalmente all'ambito "Grandezze e misure", coinvolge le seguenti risorse cognitive previste dal Piano di studio della scuola dell'obbligo ticinese (DECS, 2015):

Sapere e riconoscere: Conoscere la scansione degli intervalli di tempo della vita quotidiana (ore, giorni, settimane, mesi, stagioni e anni).

Eseguire e applicare: Situarsi nei tempi della vita quotidiana, nella loro ciclicità e nella ricorsività dei suoi eventi significativi.

\subsection{Fasi della sperimentazione e prime scoperte}

\subsubsection{Prima fase: analisi in entrata delle competenze percettive e di stima}

La prima fase del lavoro prevedeva I'analisi delle competenze percettive e di stima degli allievi. Per sondare questo aspetto sono state svolte, in momenti distinti, due attività entrambe della durata di 15 minuti. Partendo dal presupposto che la percezione del tempo sia influenzata da diversi fattori, si è deciso di proporre due attività molto diverse tra loro, in spazi distinti, con modalità di lavoro variate. La prima attività, individuale e svolta con i bambini seduti al proprio posto, consisteva nella scrittura di un testo dettato dal docente. La seconda attività, collettiva e svolta nel cortile della scuola, consisteva invece in un gioco socio-motorio: "guardie e ladri". Alla fine delle due attività è stato sottoposto agli allievi un questionario di riflessione sui momenti svolti, accompagnati da interviste individuali semi-strutturate per approfondire gli aspetti emersi dai questionari.

I questionari erano strutturati in due parti: nella prima parte è stato chiesto agli allievi di scegliere da un elenco una o più sensazioni e stati d'animo che descrivessero quanto vissuto durante l'attività, in modo da favorire una prima riflessione sull'esperienza vissuta.

Nella seconda parte del questionario sono state poste ai bambini tre domande aperte: la prima voleva sondare com'era stata percepita l'attività dagli allievi e per quale motivo era stata accolta in un determinato modo, lasciando la liberta di argomentare a piacere; la seconda voleva invece analizzare nel dettaglio le sensazioni personali vissute dai bambini durante l'attività; infine veniva chiesto di stimare la durata dell'attività svolta, in modo da ottenere una prima indicazione delle capacità di stima in entrata dei bambini (Figura 2). 
Figura 2

Esempio di questionario

completato alla fine

dell'attività socio-motoria.

\section{QUESTIONARIO}

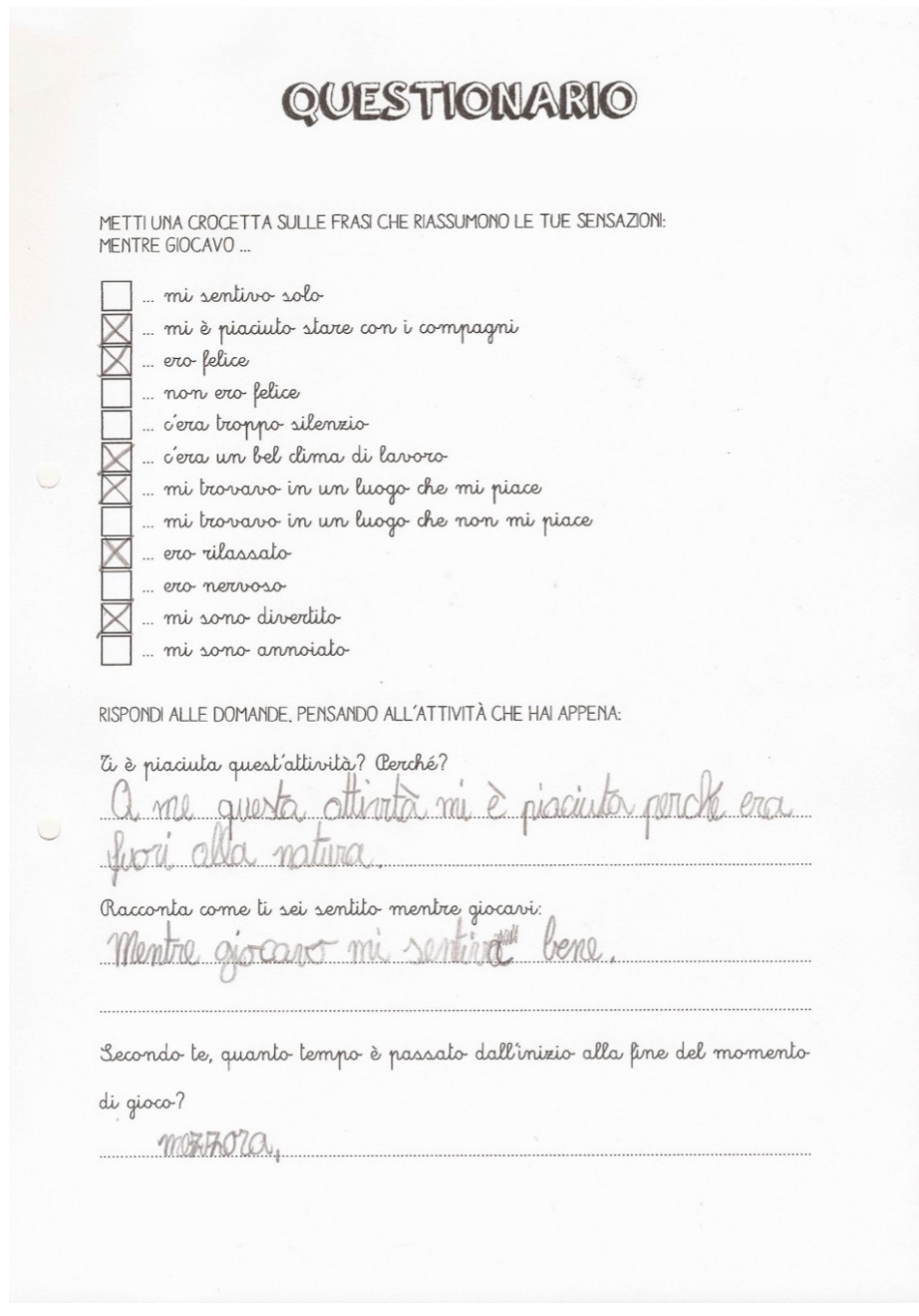

I risultati emersi da questa prima fase hanno mostrato che l'attività di scrittura è stata sottostimata da quasi la metà degli allievi, mentre il gioco "guardie e ladri" è stato sovrastimato da 13 allievi su 19 presenti. Questo significa che in generale l'attività di scrittura è stata percepita come più corta rispetto a quella di gioco. È importante notare come la totalità degli allievi abbia apprezzato l'attività di gioco, mentre 2 allievi abbiano affermato di non aver gradito l'attività di scrittura. L'ipotesi iniziale secondo la quale gli allievi avrebbero sottostimato la durata delle attività che hanno apprezzato e sovrastimato quella delle attività che hanno apprezzato meno sembrerebbe dunque rivelarsi, a questo stadio, almeno in parte infondata. In queste prime due attività i commenti degli allievi hanno permesso di riconoscere già alcune variabili, che Piaget ha categorizzato come "fattori periferici", che influenzano la percezione del tempo, quali per esempio lo sforzo, la difficoltà dell'azione svolta o l'interesse dimostrato dagli allievi per l'attività.

Potremmo interpretare i dati raccolti nella maniera seguente: i bambini tendono a stimare con più accuratezza le durate di attività di tipo "scolastico" che svolgono regolarmente e che implicano un minor numero di cambiamenti. Invece, la durata di attività meno usuali, durante le quali gli allievi si trovano in contesti alternativi e che comportano una certa fatica fisica, risultano più difficili da essere stimata. Questa ipotesi è parzialmente confermata da T., che durante il colloquio ha affermato: «Quando siamo andati fuori a giocare abbiamo corso e fatto tante cose, mi sembra- 
va che il tempo non passava mai perché non finiva il gioco».

È interessante notare come un solo bambino su 19 abbia dimostrato durante il colloquio di avere già un'idea in merito ai fattori che influenzano la nostra percezione del tempo, affermando che: «(...) di solito quando ti diverti passa più il tempo, e lì [durante il gioco] mi sono divertito ma il tempo è durato comunque tanto». L'allievo in questione sembrerebbe dunque possedere una certa consapevolezza del fatto che le sensazioni personali influiscono sulla percezione del tempo.

Soltanto un'allieva è stata in grado di riconoscere che le due attività erano della stessa durata, sottostimandole entrambe. Questo è un aspetto significativo che dimostra quanto sia difficile per un allievo di terza elementare, ma non solo, riconoscere senza il supporto di strumenti di misurazione l'egual durata di due attività organizzate in contesti diversi e con modalità differenti.

\subsubsection{Seconda fase: il quaderno del tempo}

Nella seconda fase della sperimentazione, a cadenza regolare, i bambini sono stati chiamati a riflettere sulla durata di alcune attività scolastiche. Al fine di mettere i bambini in situazioni diverse, è stato importante variare attività, modalità di lavoro e spazi. Per questo motivo le attività proposte sono state le seguenti:

- esercitazione individuale di francese, svolta al proprio posto, utilizzando un quaderno didattico;

- ascolto di due capitoli del libro "Matilde" di Roald Dahl, svolto nell'angolo biblioteca con i bambini seduti sul tappeto;

- attività di misurazione del perimetro del piano di appoggio del banco utilizzando strumenti di misura non convenzionali, quali il cubito o la spanna, svolta a coppie in varie zone dell'aula;

- esercizio di scrittura, svolto individualmente al proprio posto;

- attività di lettura individuale svolta al proprio posto, seguita da un momento di confronto e discussione sul testo appena letto realizzata a grande gruppo, con i bambini seduti in cerchio;

- allenamento delle moltiplicazioni, svolto a gruppi di 3 bambini distribuiti nell'aula;

- attività di disegno di un'esperienza vissuta a scuola, realizzata individualmente al proprio posto, con sottofondo musicale.

Tutti i momenti erano così organizzati: i bambini cominciavano l'attività al segnale del docente, svolgevano un determinato compito e, una volta annunciata la fine, riflettevano sulla durata dell'attività e sulle sensazioni provate, rispondendo alle domande presenti sul quaderno del tempo (Figura 3).

La modalità di lavoro proposta si basa sul seguente schema di lavoro:

- attività scolastica;

- riflessione, stima della sua durata;

- confronto tra stima e durata effettiva;

- nuova riflessione sul confronto tra stima e durata effettiva.

A differenza del formulario iniziale, il quaderno del tempo era strutturato in maniera più aperta, per garantire ai bambini la possibilità di esprimere le loro sensazioni senza essere influenzati da domande a risposta chiusa o a crocette. Ogni pagina del quaderno era strutturata come segue:

1. titolo o nome dell'attività svolta;

2. stima della durata dell'attività;

3. grado di apprezzamento dell'attività; 
La percezione e la stima del tempo in bambini di terza elementare: dall'intuizione alla consapevolezza dell'aspetto soggettivo / Carlo Mina

4. motivazioni relative al grado di apprezzamento dell'attività;

5. descrizione delle sensazioni provate durante l'attività;

6. motivazioni alla base delle sensazioni provate durante l'attività;

7. durata effettiva dell'attività (dato fornito dal docente);

8. confronto tra stima e durata effettiva;

9. spiegazione dell'allievo dell'eventuale incongruenza tra stima e durata effettiva.

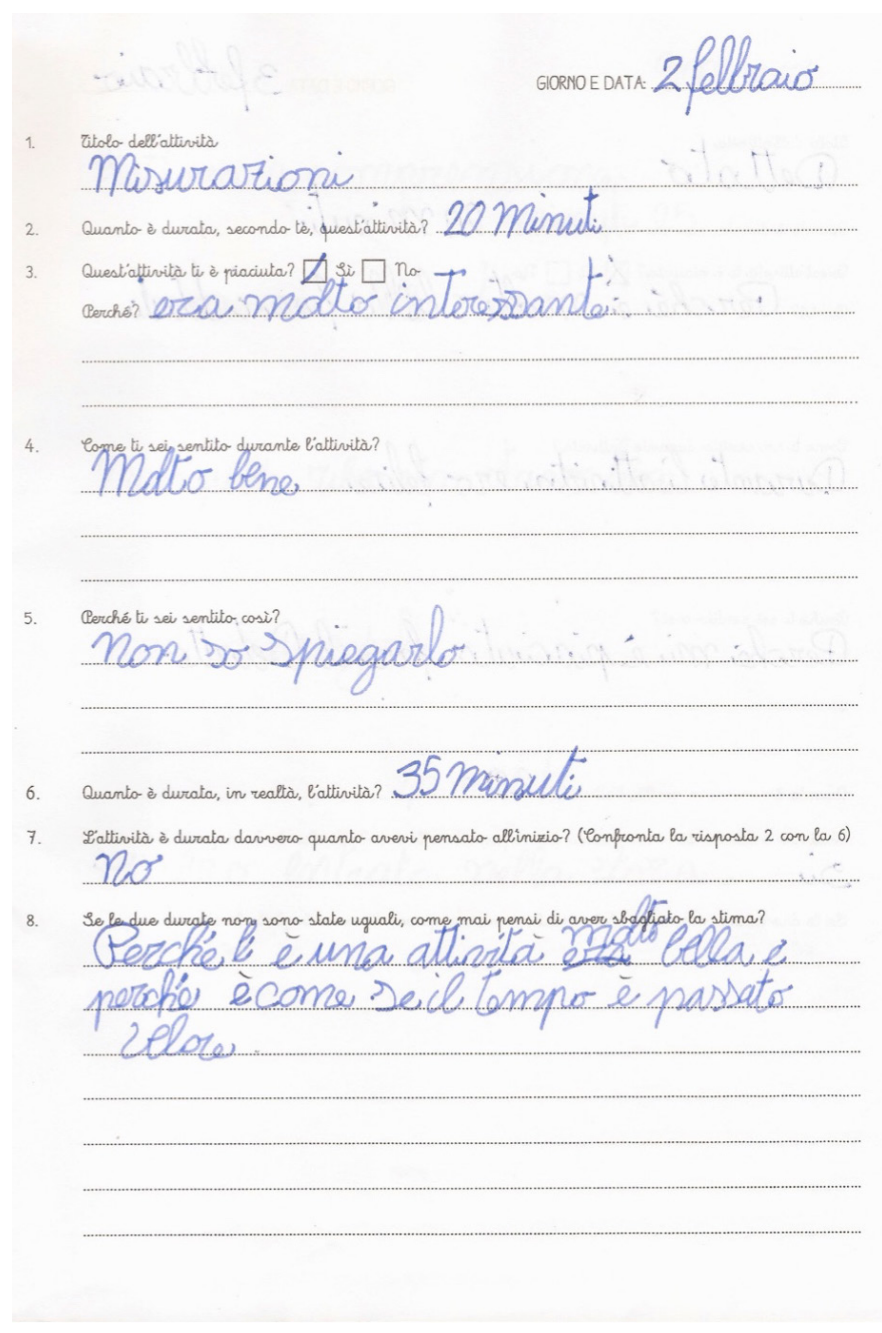

Lo scopo di questa struttura era quello di permettere ai bambini, in una prima fase, di ragionare a caldo sulla durata dell'attività svolta e sulle sensazioni vissute, lasciando loro la libertà di esprimersi in maniera piuttosto aperta. A fine riflessione veniva comunicata agli allievi la durata effettiva dell'attività, che è sempre stata misurata con strumenti diversi (clessidra ad acqua o a sabbia, orologio digitale o analogico, cronometro ecc.), e veniva chiesto loro di riflettere sull'eventuale errore di stima commesso e sulla personale interpretazione delle motivazioni alla base di queste discrepanze di stima.

A metà percorso è stato proposto un momento di discussione e messa in comune, durante il quale i bambini hanno potuto confrontarsi sul tema del tempo e su quanto emerso dalle prime riflessioni. Questa fase è stata fondamentale per favorire una presa di coscienza dell'aspetto soggettivo anche in allievi che, pur avendoci ragiona- 
La percezione e la stima del tempo in bambini di terza elementare: dall'intuizione alla consapevolezza dell'aspetto soggettivo / Carlo Mina

to, non avevano ancora raggiunto una reale consapevolezza. Di seguito è riportato un estratto della discussione che esemplifica il tipo di confronto avvenuto in classe:

A1: «Quando fai le cose e ti diverti sembra che le cose passano molto più lentamente».

A2: «Ma no!»

A1: «...eh molto più veloce, e invece se ti annoi passano più lentamente. Per esempio, se leggi un libro che non ti diverti dici che è passato un'ora e invece sono passati 10 minuti».

A3: «Però magari a me è piaciuto e a N. non è piaciuto. Quindi per lui è durato un'ora, per me è durato 5 minuti ma in verità è durato 10».

Ins.: «Quindi mi state dicendo che ognuno sente il tempo in maniera diversa?»

A1: «È diverso per tutti, dipende».

Andando a confrontare i dati ottenuti dalle attività proposte prima e dopo la discussione di metà percorso, emerge che la percentuale di bambini che sono riusciti a riflettere in maniera introspettiva è aumentata a seguito di tale discussione (Figura 4). Questo dato risulta interessante poiché consente di comprendere quanto il confronto tra allievi abbia permesso ad alcuni bambini di entrare in contatto con le idee dei compagni, sviluppando così maggiore coscienza di aspetti temporali che prima non riuscivano a cogliere.

Figura 4

Dati ottenuti dalle attività proposte prima e dopo la discussione di metà percorso.

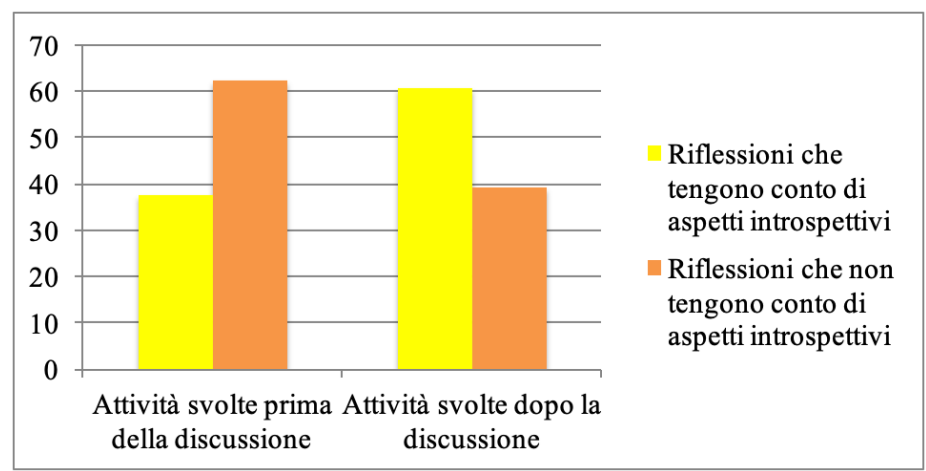

L'analisi dei dati raccolti attraverso il "quaderno del tempo", completato dopo aver realizzato le diverse attività, ha evidenziato i seguenti risultati: le 7 attività proposte durante il percorso sono state apprezzate quasi nel $90 \%$ dei casi. Le attività sono state quindi accolte perlopiù positivamente e dunque il confronto con le attività che hanno riscosso meno successo può fornire unicamente indicazioni puntuali. Analizzando il rapporto tra errore di stima della durata e livello di apprezzamento dell'attività, possiamo notare come le attività che sono piaciute agli allievi sono state sottostimate in 45 casi su 116; sono state sovrastimate in 54 casi e sono state stimate correttamente in 17 casi su 116. Invece, le attività che non sono piaciute sono state sottostimate in 12 casi su 16; sono state sovrastimate in 3 casi e sono state stimate correttamente in 1 solo caso su 16 . Contrariamente alle aspettative, dall'analisi di questi dati sembrerebbe che la maggior parte dei bambini tenda a sovrastimare la durata delle attività che hanno apprezzato e a sottostimare la durata delle attività che non hanno considerato positivamente. Questo dato ci consente di intuire che i bambini non sono influenzati unicamente dalle loro sensazioni e dal loro interesse 
per l'attività svolta, ma da una serie di fattori evidentemente più ampi. Non sono dunque soltanto il divertimento o la noia ad influire sulla percezione tempo e sulla stima delle durate negli allievi. I dati emersi dalle tabelle sono stati analizzati cercando di individuare e categorizzare i fattori che influenzano la percezione del tempo nei bambini (Allegato 1).

Analizzando questi dati ci si rende conto della presenza di 3 tipi di motivazioni che i bambini hanno fornito con più frequenza, indipendentemente dall'apprezzamento o meno dell'attività:

- tipologia dell'attività (p. es.: "mi piacciono i dettati", "mi piace disegnare" ecc.);

- motivazioni intrinseche e personali (p. es: "perché per me era bella", "perché non mi è piaciuta" ecc.);

- condizioni e clima di lavoro (p. es.: "perché eravamo fuori all'aria aperta", "perché c'era molto silenzio" ecc.).

|l $76 \%$ delle motivazioni relative ad attività che sono piaciute e addirittura il 95\% di quelle relative ad attività che non sono state apprezzate sono riconducili a queste 3 categorie. Ciò significa che queste sono le principali motivazioni che spingono un allievo ad apprezzare o meno una determinata attività e probabilmente, in maniera implicita, questi sono anche alcuni dei fattori che influiscono la percezione del tempo degli allievi.

Osservando le variabili che influenzano la percezione del tempo individuate dai diversi autori (vedi Tabella 1), notiamo come alcune delle tipologie di motivazioni dei bambini siano compatibili con le categorie di fattori periferici. Infatti, le motivazioni relative alla tipologia dell'attività potrebbero rientrare nella categoria dei fattori periferici della prevedibilità, dell'interesse e della soddisfazione; le motivazioni intrinseche e personali potrebbero essere inserite nei fattori periferici dell'interesse e della soddisfazione; mentre le motivazioni relative a condizioni e clima di lavoro potrebbero formare una nuova categoria di fattori periferici.

\subsubsection{Valutazione del percorso e discussione finale}

Nell'ultima fase del percorso si è proposto un questionario di valutazione di quanto svolto, nel quale ogni allievo ha cercato di spiegare la valenza formativa di quanto fatto e ha provato ad esplicitare quanto appreso.

Il questionario di bilancio chiedeva innanzitutto di dare un giudizio al percorso svolto, attribuendogli poi tre aggettivi che lo potessero descrivere: questo ci ha permesso di capire se il lavoro svolto è stato apprezzato o meno. In seguito si è voluta sondare I'utilità del percorso vissuto dagli allievi, chiedendo loro cosa hanno imparato e a cosa è servito il lavoro, in modo da capire se c'è stata una presa di coscienza alla fine del percorso. Le domande successive sono invece state poste per spingere i bambini a ragionare in maniera metacognitiva sui processi messi in atto durante le attività di stima. Si è infine chiesto di elencare tutti gli strumenti per la misura del tempo conosciuti dai bambini, così da capire quanto gli strumenti utilizzati durante il percorso avessero lasciato un'impronta.

Ciò che è emerso da questi questionari è poi stato condiviso con l'intera classe durante una discussione che ha permesso ai bambini di argomentare le proprie posizioni. 
Figura 5

Il bilancio finale redatto da un allievo.
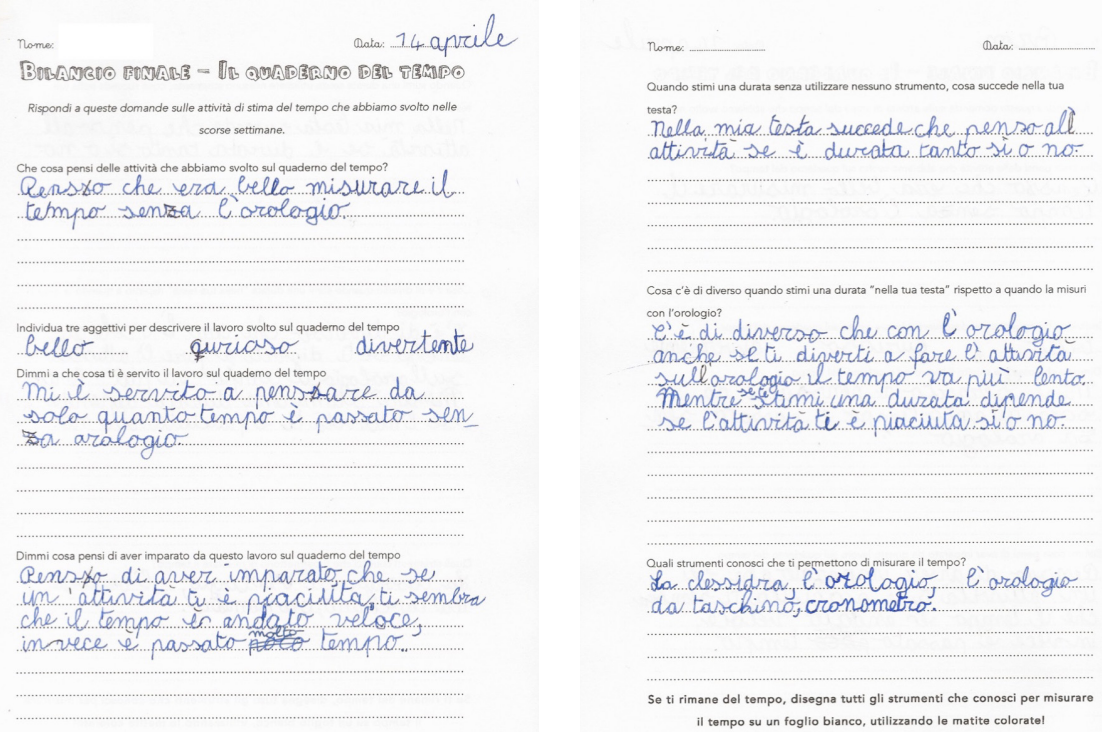

Dai dati raccolti emerge che il percorso è stato accolto in maniera generalmente positiva dagli allievi: 17 bambini hanno infatti affermato di aver apprezzato le attività sul quaderno del tempo; 2 bambini hanno affermato di non averle apprezzate e 1 bambino ha affermato di averne apprezzate alcune e altre meno. Gli allievi che hanno riconosciuto di aver imparato nuove cose durante il percorso svolto sul tempo sono 13 su 20.4 allievi hanno affermato di non aver appreso nulla da queste attività, i restanti 3 bambini hanno dato risposte generiche, scrivendo frasi come "credo di aver imparato il tempo» o "ho imparato a tenere nota e a distinguere il tempo che passa».

Dal questionario finale emerge inoltre come 13 bambini su 20, a fine percorso, siano stati in grado di riflettere in maniera introspettiva sulla percezione del tempo. Di questi, 9 allievi hanno dato spiegazioni relative all'influenza che hanno le sensazioni di noia e divertimento sulla percezione del tempo, sostenendo per esempio che «il tempo che passa, se c'è qualcosa di bello passa più veloce, invece se l'attività non ti piace non passa più». I restanti 4 allievi hanno dato spiegazioni un po' meno dettagliate, affermando per esempio che "Quando stimo una durata nella mia testa non mi sento aiutata. Ad esempio se passano 15 minuti magari per me ne sono passati 30»; "Con l'orologio sono avvantaggiato perché so la durata giusta, invece con la mia testa è più difficile. Magari io sbaglio, invece sull'orologio non sbaglio». Gli esiti degli studi di Piaget e di Cardaci, che riconoscono nel livello di soddisfazione del vissuto e nel tempo dell'attesa e dell'interesse dei fattori che influenzano la percezione del tempo, sono dunque almeno parzialmente confermati da questa sperimentazione.

Possiamo infine notare come il 95\% degli allievi abbia riconosciuto una differenza tra tempo fisico e tempo psicologico, mentre 1 sola allieva ha affermato che tra questi non c'è distinzione. Tra gli allievi che hanno riconosciuto una differenza tra tempo psicologico e tempo fisico, in 6 hanno fatto riferimento al fatto che la mente è influenzata da fattori soggettivi, mentre l'orologio non è influenzabile ed è preci- 
so; 2 allievi hanno affermato che I'orologio è preciso mentre la mente no; 7 allievi hanno affermato che con l'orologio è possibile vedere il tempo, mentre nella mente non si vede ed è necessario riflettere e fare delle stime; 2 allievi hanno affermato che utilizzare l'orologio è più facile, mentre stimare le durate è più difficile e altri 2 allievi hanno semplicemente affermato che l'orologio «è meglio». I dati raccolti sono sintetizzati nella tabella presente nell'Allegato 2 .

I dati raccolti nelle tre fasi di questo lavoro, pur non essendo rappresentativi, mettono in discussione l'opinione di Piaget (1979), secondo il quale «nei più piccoli, il carattere egocentrico, ossia immediato e irreversibile, del pensiero è un ostacolo ad ogni introspezione» (p. 266). I dati raccolti dimostrano infatti che la maggior parte degli allievi di terza elementare, se messi a confronto con le idee dei compagni e spronati a riflettere sul loro operato, sono in grado di sviluppare delle interessanti riflessioni a carattere introspettivo. Ė pure opportuno ipotizzare che, proponendo un lavoro di questo tipo in maniera continuata per tutto il percorso scolastico degli allievi, tali capacità di riflessione introspettiva possano migliorare.

\section{Conclusioni}

I risultati ottenuti da questa sperimentazione hanno evidenziato come un lavoro vissuto dagli allievi sulla stima e sulla riflessione relativa al tema tempo abbia portato ad una presa di coscienza riguardo ai fattori soggettivi che influenzano la nostra percezione. Ciò dimostra che è possibile trattare questo tema già con allievi di scuola elementare, ottenendo risultati concreti.

L'analisi dei dati raccolti durante questi momenti di lavoro sulla percezione e sulla stima del tempo ha permesso di appurare, contrariamente a quanto ipotizzato inizialmente, come le capacità di stima delle durate degli allievi non siano evolute in maniera sostanziale. Tuttavia, questo percorso ha permesso alla maggior parte degli allievi di prendere coscienza di alcuni fattori che influenzano la percezione del tempo e di sviluppare una maggior consapevolezza sull'aspetto soggettivo della percezione. Questo è stato possibile anche grazie ai momenti di confronto e di discussione, strutturati partendo da quanto emerso nelle riflessioni dei bambini, che si sono confermati dei potenti mezzi di condivisione e di costruzione di senso. Favorire il confronto tra pari in un contesto di riflessione sulla percezione del tempo risulta dunque importante in quanto, come dimostrato in questo lavoro, permette ai bambini di prendere coscienza, attraverso le esperienze dei compagni, del rapporto tra sensazioni provate e percezione temporale.

Al fine di proporre un percorso più efficace sarebbe ovviamente utile dedicare un lasso di tempo maggiore alle attività di riflessione su questo tema. Nella migliore delle ipotesi, un percorso dilatato sull'intero anno scolastico sarebbe la soluzione ideale. Inoltre, per non limitare i momenti di riflessione all'utilizzo del quaderno del tempo, si potrebbero proporre anche altre attività legate alla percezione del tempo, in modo da ampliare e rendere più completo il percorso dal punto di vista didattico. Alcuni esempi potrebbero essere momenti di riflessione su esperienze vissute dai compagni e sulle relative stime legate al tempo; situazioni problema legate all'influenza della percezione del tempo nel vissuto dei bambini; scoperta di vari strumenti di misura del tempo e relative modalità di funzionamento; la lettura dell'orologio; eccetera. 
La percezione e la stima del tempo in bambini di terza elementare: dall'intuizione alla consapevolezza dell'aspetto soggettivo / Carlo Mina

L'utilizzo di vari strumenti di misura del tempo potrebbe poi venir esteso a tutti i momenti della vita di classe. I bambini potrebbero utilizzare la clessidra durante il lavoro autonomo, oppure il cronometro per stabilire la durata delle attività laboratoriali, in modo da prendere dimestichezza con questi strumenti e osservare concretamente lo scorrere del tempo fisico con modalità diverse. Favorendo queste esperienze su un esteso lasso di tempo, è ipotizzabile un miglioramento delle capacità di stima delle durate degli allievi, cosa che non è emersa in questo lavoro, forse a causa del limitato orizzonte temporale su cui si è protratta.

A questo punto non resta che sperimentare in classe: le riflessioni dei bambini sapranno sicuramente accendere l'entusiasmo e la curiosità di qualsiasi docente.

\section{Bibliografia}

Aiolfi, A. M. (2009). Numeri, spazio e tempo. Attività di scienze per fare matematica. Roma: Carocci editore.

Bartolini Bussi, M. G. (2008). Matematica. I numeri e lo spazio. Azzano San Paolo: Junior edizioni.

Calvani, A. (1988). Il bambino, il tempo, la storia. Scandicci: La Nuova Italia editrice.

Cardaci, M. (2004). Mi cambierebbe 25 minuti? Roma: Francesco Bannò Editore.

DECS. (2015). Piano di studio della scuola dell'obbligo ticinese. Disponibile in http://www. pianodistudio.ch/sites/default/files/pdf/Piano di studio della scuola dell obbligo tici nese COMPLETO.pdf (consultato l'11.01.2019).

Fraisse, P., \& Piaget, J. (1975). Trattato di psicologia sperimentale. La percezione. Torino: Einaudi.

Fraisse, P., Halberg, F., Lejeune, H., Michon, A., Montangero, J., Nuttin, J., \& Richelle, M (1979). Du temps biologique au temps psychologique. Parigi: Presses Universitaires de France.

Karmiloff-Smith, A. (1992). Oltre la mente modulare. Una prospettiva evolutiva sulla scienza cognitiva. Bologna: il Mulino.

Lahman, M.K.E. (2008). Always othered. Journal of early childhood research, 6(3), 281-300.

Lakoff, G., \& Núñez, E.R. (2000). Da dove viene la matematica. Come la mente embodied dà origine alla matematica. Torino: Bollati Boringhieri.

Levin, I. (1982). The nature and development of time concepts in children: the effects of interfering cues, in: Friedman, W.J. The developmental psychology of time. New York: Academic Press.

Mauthner, M. (1997). Methodological aspects of collecting data from children: lessons from three research project. Children \& society, 11, 16-28.

Moore, T., McArthur, M., \& Noble-Carr, D. (2008). Little voices and big ideas: lessons learned from children about research. International journal of qualitative methods, 7(2), 77-91.

Pea, B. (2001). Matematica nella scuola di base. I concetti dello spazio e del tempo nella scuo- 
La percezione e la stima del tempo in bambini di terza elementare: dall'intuizione alla consapevolezza dell'aspetto soggettivo / Carlo Mina

la materna e nel primo ciclo della scuola di base. Volume 1. Brescia: Società editrice Vannini.

Petter, G. (1978). Lo sviluppo mentale nelle ricerche di Jean Piaget. Firenze: Giunti Barbèra.

Piaget, J. (1979). Lo sviluppo della nozione di tempo nel bambino. Firenze: La Nuova Italia editrice.

Punch, S. (2002). Research with children: the same or different from research with adults Childhood?, 9, 321-341.

Sandri, P. (2008a). La didattica del tempo convenzionale. Riflessioni e percorsi per la scuola dell'infanzia e la scuola primaria. Milano: Franco Angeli.

Sandri, P. (2008b). Rappresentazioni temporali e deficit intellettivo lieve. Proposte didattiche per la scuola primaria. Milano: Franco Angeli.

Skutina, V., \& Sacré, M.J. (1989). Dove abita il tempo. Milano: Edizioni Arka.

Saurer, T. (2014). Piaget, Einstein, and the concept of time. Disponibile in: http://philsci-ar chive.pitt.edu/10637/1/damerow.pdf (consultato il 03.04.2015).

Autore/Carlo Mina

Istituto Scolastico di Locarno - Sede di Solduno, Svizzera

carlo.mina@edu.ti.ch 